\title{
REGULAR FUNCTIONS OF RESTRICTED GROWTH AND THEIR ZEROS IN TANGENTIAL REGIONS
}

BY

\author{
C. N. LINDEN
}

\begin{abstract}
For a given function $k$, positive, continuous, nondecreasing and unbounded on $[0,1)$, let $A^{(k)}$ denote the class of functions regular in the unit disc for which $\log |f(z)|<k(|z|)$ when $|z|<1$. Hayman and Korenblum have shown that a necessary and sufficient condition for the sets of positive zeros of all functions in $A^{(k)}$ to be Blaschke is that

$$
\int_{0}^{1} \sqrt{ }(k(t) /(1-t)) d t
$$

is finite. It is shown that the imposition of a further regularity condition on the growth of $k$ ensures that in some tangential region the zero set of each function in $A^{(k)}$ is also Blaschke.
\end{abstract}

1. Introduction. Let $k$ be a positive, continuous, nondecreasing unbounded function defined on the interval $(0,1)$. W. K. Hayman and B. Korenblum [1] have denoted by $A^{(k)}$ the class of functions $f$, regular in the unit disc $D(0,1)$, and satisfying the inequality

$$
\log |f(z)|<k(|z|) \quad(|z|<1) .
$$

The class $A^{(k)}$ is said to be Shapiro-Shields if and only if the set of positive zeros $\left\{\rho_{n}(f)\right\}$ of each nonconstant function $f$ in $A^{(k)}$ is a Blaschke sequence, that is

$$
\sum_{n}\left(1-\rho_{n}(f)\right)<\infty
$$

for each $f$ in $A^{(k)}$. Thus the principal result of Hayman and Korenblum appears as follows.

THEOREM A. A necessary and sufficient condition for $A^{(k)}$ to be Shapiro-Shields is

$$
J(k)=\int_{0}^{1} \sqrt{ }(k(t) /(1-t)) d t<\infty .
$$

The class of functions $k$ that satisfy (1.3) has no extreme elements in the sense that for any given $k$ there is a positive, continuous, nondecreasing, unbounded function $u$ such that $J(k u)<\infty$. For this reason, and in spite of the fact that the theorem of Hayman and Korenblum is stated in terms of a necessary and sufficient condition, something more than (1.2) might be expected concerning the zero distribution of a function $f$ in a given Shapiro-Shields class $A^{(k)}$. Hayman and Korenblum have indicated a result in this direction by pointing out that condition (1.3) is, in fact,

Received by the editors September 2, 1981.

1980 Mathematics Subject Classification. Primary 30D35; Secondary 30D50.

(C) 1983 American Mathematical Society 0002-9947/82/0000-0137/\$03.00 
sufficient to ensure that for any nonconstant function $f$ in $A^{(k)}$, and any Stolz domain $S$ with a vertex on the unit circle, the zero set $\left\{z_{n}(f, S)\right\}$ of $f$ in $S$ is Blaschke in the sense that

$$
\sum_{n}\left(1-\left|z_{n}(f, S)\right|\right)<\infty .
$$

The aim of this paper is to show that extra conditions, applied only to the regularity of growth of $k$, are sufficient to ensure that the sets of zeros of functions $f$ in $A^{(k)}$, counted over certain tangential regions, are still necessarily Blaschke. Two versions of the smoothness conditions are given. The first and more restrictive version relates the tangential regions directly to the function $k$ as follows.

THEOREM 1. Let $k$ be a positive, unbounded function, continuous on $(0,1)$ and satisfying (1.3). Further, let

$$
k(t)(1-t) \text { be monotonic decreasing }
$$

and, for some constant $d$, let

(1.6) $k(t)(1-t)(\log 1 /(1-t))^{2 d}$ be monotonic increasing as $t$ increases to 1 .

Then if $f$ is a nonconstant function in $A^{(k)}$ there are constants $m$ and $L$ such that the set of zeros of $f$ in

$$
\left\{z:\left|\arg \frac{z}{\xi}\right|<L_{\sqrt{ }}((1-|z|) / k(|z|)),|\xi-z|<m\right\}
$$

is a Blaschke sequence. Moreover the Blaschke sum for the zeros of $f$ in (1.7) has a bound which is independent of $\xi$ on $\partial D(0,1)$.

It should be noted that the conditions satisfied by $k$ ensure that

$$
\lim _{t \rightarrow 1-} k(t)(1-t)=0,
$$

so that the region $(1.7)$ is tangential to $\partial D(0,1)$. The constant $L$ will be found to depend on $k$, and $m$ will be any constant chosen small enough to ensure that $\partial D(0,1)$ intersects the boundary of $(1.7)$ only at $\xi$.

Theorem 2, which follows, indicates that even if (1.6) itself is not assumed then a suitable majorant can still be found for $k$. Thus we obtain a result that leads to the fact that functions in an extended class $A^{(k)}$ also have Blaschke sequences of zeros in certain tangential regions. However the relation of $k$ to the tangential regions is not so straightforward in this case.

THEOREM 2. Let $k$ be a positive, unbounded function, continuous on $[0,1)$, and satisfying (1.3). Then if (1.5) is satisfied as $t$ increases to 1 , there is a positive, unbounded function $\nu$, continuous on $[0,1)$, such that $k<\nu, J(\nu)<\infty$, while $\nu(t)(1-t)$ decreases and $\nu(t)(1-t)(\log (1 /(1-t)))^{4}$ increases as $t$ increases to 1 .

COROLlary 1. If $k$ is a positive, unbounded function, continuous on $(0,1)$, and such that (1.3) and (1.5) are both satisfied, then for each nonconstant function $f$ in $A^{(k)}$ and each point $\xi$ on $\partial D(0,1)$ there is a tangential region $T_{\xi}$ in $D(0,1)$ such that $\bar{T}_{\xi} \cap \partial D(0,1)$ is the single point $\xi$, and the set of zeros of $f$ in $T_{\xi}$ is a Blaschke sequence. Further, we may take $T_{\xi}$ to be $T_{1}$, rotated about 0 through an angle equal to arg $\xi$. 
2. Preliminaries. Theorem 1 will be proved by the adaptation of a method used earlier [2] to obtain some inequalities for real harmonic functions. The main tool used was the following theorem.

THEOREM B. Let $\chi$ be a positive, continuous, nondecreasing, unbounded function defined on an interval $\left(x_{0}, \infty\right)$ such that

$$
F(r)=\int_{r}^{\infty} \frac{d t}{t \chi(t)}<\infty \quad\left(r \geqslant x_{0}\right) .
$$

For some positive number $d$, let $(\log x)^{d} / \chi(x)$ increase as $x$ increases, and let

$$
U(z)=\int_{x_{0}}^{\infty} \sqrt{ }\left(\frac{z}{t}\right) \frac{F(t)}{t+z} d t .
$$

If $0<\delta<\pi$ then $U$ is regular on the set

$$
G=\left\{r e^{i \theta}: r>0,|\theta| \leqslant \pi-\delta\right\}
$$

and

$$
\begin{gathered}
\Re U\left(r e^{i \theta}\right)=\pi F(r)(1+B(r, \theta)), \\
\mathscr{G U}\left(r e^{i \theta}\right)=\frac{-A(r, \theta) \sin \frac{1}{2} \theta}{\chi(r)},
\end{gathered}
$$

where $B(r, \theta)$ and $\log A(r, \theta)$ are bounded on $G$ and $\lim _{r \rightarrow \infty} B(r, \theta)=0$ uniformly when $\theta \in[-\pi+\delta, \pi-\delta]$.

We first transform the context of Theorem 1 into one more suitable for the application of Theorem B. Thus we define

$$
\chi(t)=\left(\frac{t}{k(1-1 / t)}\right)^{1 / 2} \quad(t>1),
$$

noting that if $k$ satisfies the hypothesis of Theorem 1 then $\chi$ satisfies the hypothesis of Theorem B. The function $U$ defined by (2.2) is regular on some set

$$
H=\left\{z:|\arg z| \leqslant \frac{1}{2} \pi,|z| \geqslant r_{0}\right\} .
$$

Since (2.1), (2.4) and (2.5) imply that $\lim _{z \rightarrow \infty} U(z)=0$ uniformly in $H$ we may choose $r_{0}$ so that

$$
|U(z)|<\frac{1}{4} \quad(z \in H) .
$$

Without loss of generality we may also assume that $x_{0}$ is chosen so that

$$
F(t)<\frac{1}{8} \quad\left(t \geqslant x_{0}\right) .
$$

It has been shown [2] that the function $\phi$ defined by the relation

$$
\phi(z)=1-\frac{1-U(z)}{z} \quad(z \in H)
$$

is regular, and maps $H$ onto a subset of $D(0,1)$. The function $g$, where

$$
g(z)=f(\phi(z)) \quad(z \in H)
$$


is also regular, and (1.1) and (2.6) imply that

$$
\log |g(z)|<\frac{K|z|}{\chi(|z|)} \quad(z \in H)
$$

for some constant $K$. We now need to extend our knowledge of $\phi$ beyond these known facts to show that $\phi$ is also schlicht in $H$ for some positive number $r_{0}$.

If $\phi$ is not schlicht in $H$ then there are two numbers $\omega=r e^{i \theta}$ and $W=R e^{i \Psi}$ in $H$ such that $(1-U(\omega)) / \omega=(1-U(W)) / W$, that is

$$
1-U(\omega)=\omega\left(\frac{U(W)-U(\omega)}{\omega-W}\right) .
$$

Without loss of generality we may assume that $r_{0} \leqslant r \leqslant R$. The definition (2.2) implies that

$$
\frac{U(W)-U(\omega)}{\omega-W}=\frac{I}{\sqrt{ } \omega+\sqrt{ } W}
$$

where

$$
I=\int_{x_{0}}^{\infty} \frac{F(t)(\sqrt{ }(\omega W)-t)}{\sqrt{t}(t+\omega)(t+W)} d t .
$$

However, since $|\theta| \leqslant \frac{1}{2} \pi$ and $|\Psi| \leqslant \frac{1}{2} \pi$ we have

$$
\left|\frac{\sqrt{ }(\omega W)-t}{t+W}\right|^{2}=\frac{t^{2}+r R-2 t_{\sqrt{ }}(r R) \cos \frac{1}{2}(\theta-\Psi)}{t^{2}+R^{2}+2 t R \cos \Psi} \leqslant \frac{t^{2}+r R}{t^{2}+R^{2}} \leqslant 1,
$$

and

$$
|t+\omega|^{2}=t^{2}+2 t r \cos \theta+r^{2} \geqslant \frac{1}{2}(t+r)^{2} .
$$

Thus (2.8) implies that

$$
|I| \leqslant \int_{x_{0}}^{\infty} \frac{\sqrt{ } 2 F(t)}{\sqrt{ } t(r+t)} d t<\int_{0}^{\infty} \frac{1}{4_{\sqrt{ }(2 t)(r+t)}} d t=\frac{\pi}{4_{\sqrt{ }(2 r)}(2 r} .
$$

Since we also have

$$
|\sqrt{ } \omega+\sqrt{ } W|^{2}=r+R+2 \sqrt{ }(r R) \cos \frac{1}{2}(\theta-\Psi) \geqslant 2 r,
$$

we obtain

$$
\left|\omega\left(\frac{U(W)-U(\omega)}{\omega-W}\right)\right|<\frac{\pi}{8} .
$$

The inequality (2.10), together with (2.7) and (2.11), gives a contradiction. It follows that $\phi$ is schlicht in $H$ for some positive number $r_{0}$.

3. The proof of Theorem 1. We are now in a position to complete the proof of Theorem 1. Suppose, without loss of generality, that $\zeta=1$. Since the function $g$ is being regular on the set $H$ we have, by a standard application of Carleman's theorem [3, pp. 130-132],

$$
\sum_{n} \frac{\cos \theta_{n}}{\rho_{n}}<\infty
$$


where $\left\{\rho_{n} e^{i \theta_{n}}\right\}$ denotes the set of zeros of $g$ in $H$. Let $\zeta$ be the zero of $f$ corresponding to a zero $z=\rho e^{i \theta}$ of $g$ in $H$, multiple zeros being paired off in a suitable way.

If $\zeta$ belongs to the region (1.7) then

$$
|\arg \zeta|<L(1-|\zeta|) \chi(1 /(1-|\zeta|)) \text {. }
$$

We will show that this condition implies that for appropriate constants $A$ and $L$

$$
1-|\zeta|<\frac{A \cos \theta}{\rho}
$$

for sufficiently small values of $|1-\zeta|$.

Since $\zeta=\phi(z)=1-(1-U(z)) / z$ we may put

$$
\zeta=s e^{i \Psi}, \quad z=\rho e^{i \theta}, \quad 1-U(z)=t e^{i \omega}
$$

to obtain $s e^{i \Psi}=1-t e^{i(\omega-\theta)} / \rho$. Thus we have

$$
1-|\zeta|<1-|\zeta|^{2}=\frac{2 t}{\rho} \cos (\omega-\theta)-\frac{t^{2}}{\rho^{2}}<\frac{2 t}{\rho} \cos (\omega-\theta),
$$

which, together with (3.2), implies that

$$
\begin{aligned}
\frac{t}{\rho} & =|1-\zeta|<(1-|\zeta|)(1+L \chi(1 /(1-|\zeta|))) \\
& <(1-|\zeta|)\left(1+A(\log (1 /(1-|\zeta|)))^{d}\right) .
\end{aligned}
$$

It follows that $\log (1 /(1-|\zeta|))<2 \log (1 /|1-\zeta|)$ if $|1-\zeta|$ is sufficiently small. Hence we have

$$
\chi(1 /(1-|\zeta|))<2^{d} \chi(1 /|1-\zeta|)
$$

so that

$$
\begin{aligned}
\frac{t}{\rho} & <\frac{2 t}{\rho} \cos (\omega-\theta)\left(1+L 2^{d} \chi(1 /|1-\zeta|)\right) \\
& =\frac{2 t}{\rho} \cos (\omega-\theta)\left(1+L 2^{d} \chi\left(\frac{\rho}{t}\right)\right)
\end{aligned}
$$

The relation (2.5) enables us to deduce that

$$
\begin{aligned}
\cos (\omega-\theta) & =\cos \omega \cos \theta+\sin \omega \sin \theta \\
& <\cos \theta+\frac{A(\rho, \theta)}{t \chi(\rho)} \\
& <\cos \theta+\frac{2 A(\rho, \theta) \cos (\omega-\theta)\left(1+L 2^{d} \chi(\rho / t)\right)}{t \chi(\rho)} .
\end{aligned}
$$

Since $t$ tends uniformly to 1 as $\rho$ tends to $\infty$, the inequalities (3.5) enable us to deduce (3.3) for some sufficiently small positive number $L$. Thus the proof of Theorem 1 is complete. 
4. The proof of Theorem 2. It is convenient to prove Theorem 2 in the following form applicable to functions defined on $[1, \infty)$ rather than on $[0,1)$.

LEMMA 1. Let $\chi$ be a positive, continuous, monotonic increasing, unbounded function defined on $[1, \infty)$ and satisfying the condition

$$
\int_{1}^{\infty} \frac{d x}{x \chi(x)}<\infty
$$

Then there is a function $\lambda$, positive, continuous, monotonic increasing, and unbounded on $(1, \infty)$ such that $\lambda \leqslant \chi$

$$
\int_{1}^{\infty} \frac{d x}{x \lambda(x)}<\infty
$$

and $\lambda(x) /(\log x)^{2}$ decreases as $x$ increases.

The equivalence of Lemma 1 and Theorem 2 is due to the fact that if

$$
\lambda(1 /(1-t))=\frac{1}{\sqrt{ }((1-t) K(t))}
$$

then

(i) (4.2) is equivalent to

$$
\int_{0}^{1} \sqrt{ }\left(\frac{K(t)}{(1-t)}\right) d t<\infty
$$

(ii) $\lambda(x)$ increases with $x$ on $[1, \infty)$ if and only if $(1-t) K(t)$ decreases as $t$ increases on $[0,1)$,

(iii) $\lambda(x) /(\log x)^{2}$ decreases as $x$ increases on $(1, \infty)$ if and only if

$$
(1-t) K(t)(\log 1 /(1-t))^{4}
$$

increases with $t$ on $(0,1)$.

We prove Lemma 1 by first obtaining suitable bounds to the members of the sequence $\left\{\chi\left(2^{n}\right)\right\}$ and then elaborating these bounds by means of an interpolation process. Let us note that

$$
\sum_{n=1}^{\infty} \frac{1}{\chi\left(2^{n}\right)}<2 \int_{1}^{\infty} \frac{1}{x \chi(x)} d x<\infty
$$

In defining an auxiliary sequence $\left\{a_{n}\right\}$ we suppose, without loss of generality, that $a_{1}=\lim _{x \rightarrow 1} 1 / \chi(x)$ is a real number. Then we specify the inductive process

$$
a_{n+1}=\sup \left\{\frac{1}{\chi\left(2^{n+1}\right)}, \frac{(n+1) a_{n}}{n+3}\right\} \quad(n \geqslant 1) .
$$

It follows that $\left\{a_{n}\right\}$ is a decreasing sequence since either

$$
a_{n+1}=\frac{1}{\chi\left(2^{n+1}\right)} \leqslant \frac{1}{\chi\left(2^{n}\right)} \leqslant a_{n}
$$

or

$$
a_{n+1}=\frac{(n+1) a_{n}}{n+3}<a_{n} \text {. }
$$


We next show that $\sum_{n=1}^{\infty} a_{n}<\infty$. The definition (4.4) shows that either,

(iv) $a_{n+1}=1 / \chi\left(2^{n+1}\right)\left(n \geqslant n_{0}\right)$ or

(v) $a_{n+1}=(n+1) a_{n} /(n+3)\left(n \geqslant n_{0}\right)$

may hold for some integer $n_{0}$, and that if neither (iv) nor (v) holds as specified then

(vi) there are infinite sequences of integers $\{p(j)\}$ and $\{q(j)\}$ such that

$$
\begin{array}{ll}
a_{n+1}=\left(\frac{n+1}{n+3}\right) a_{n} & (p(j) \leqslant n<q(j)), \\
a_{n+1}=\frac{1}{\chi\left(2^{n+1}\right)} \quad(q(j) \leqslant n<p(j+1)) .
\end{array}
$$

Clearly $\sum_{n=1}^{\infty} a_{n}<\infty$ if (iv) or (v) holds. If (vi) holds we define

$$
A(j)=\mathbf{N}_{\cap}[p(j), q(j)), \quad B(j)=\mathbf{N}_{\cap}[q(j), p(j+1)) .
$$

The inequality (4.3) shows that $\sum_{j=1}^{\infty} \sum_{n \in B(j)} a_{n+1}<\infty$. In considering the corresponding sums when $n \in A(j)$ we define $S_{j}=\sum_{n \in A(j)} a_{n+1}$, where

$$
\begin{aligned}
S_{j} & =\sum_{n \in A(j)} \frac{(p(j)+2)(p(j)+1)}{(n+3)(n+2)} a_{p(j)} \\
& =\frac{(p(j)+1)(q(j)-p(j))}{q(j)+2} a_{p(j)} \\
& =\frac{(q(j)+1)(q(j)-p(j))}{p(j)+2} a_{q(j)} .
\end{aligned}
$$

We note, in particular, that

$$
a_{q(j)+1}=\frac{1}{\chi\left(2^{q(j)+1}\right)}, \quad a_{p(j)}=\frac{1}{\chi\left(2^{p(j)}\right)} \quad(j \in \mathbf{N}) .
$$

Let us now consider separately the cases where (a) $q(j) \leqslant 2 p(j)$ and where (b) $q(j)>2 p(j)$. If (a) holds then

$$
\begin{aligned}
S_{j} & <2(q(j)-p(j)) a_{q(j)} \leqslant \frac{2(q(j)-p(j))(q(j)+3)}{q(j)+1} a_{q(j)+1} \\
& \leqslant \frac{4(q(j)-p(j))}{\chi\left(2^{q(j)+1}\right)} \leqslant \sum_{n \in A(j)} \frac{4}{\chi\left(2^{n}\right)} .
\end{aligned}
$$

Hence $\Sigma_{j} S_{j}$, where the sum extends over all $j$ for which (a) is true, is finite.

It remains to consider $\Sigma_{j} S_{j}$, where the sum extends over all $j$ for which (b) holds. Without loss of generality, and to save some tedious reorganization, we may now assume that (b) holds for each $j$ in $\mathbf{N}$. Then, since $p(j)>q(j-1)>2 p(j-1)$, we have

$$
\begin{aligned}
S_{j} & =\frac{(p(j)+1)(q(j)-p(j))}{q(j)+2} a_{p(j)}<\frac{p(j)+1}{\chi\left(2^{p(j)}\right)} \\
& \leqslant \frac{2(p(j)-p(j-1))}{\chi\left(2^{p(j)}\right)} \leqslant \sum_{n=p(j-1)}^{p(j)-1} \frac{2}{\chi\left(2^{n}\right)}
\end{aligned}
$$


for each $j$. Thus we have proved that $\sum_{j=1}^{\infty} S_{j}$ must also converge. Hence $\sum_{n=1}^{\infty} a_{n}$ converges.

Having found a suitable sequence $\left\{a_{n}\right\}$ of real numbers which majorises the sequence of function values $\left\{1 / \chi\left(2^{n}\right)\right\}$, we now turn our attention to deducing an appropriate majorant for the function $1 / \chi$ itself. To this end we define

$$
\lambda(x)=\frac{a_{n-2} \log \left(x / 2^{n-1}\right)+a_{n-1} \log \left(2^{n} / x\right)}{a_{n-1} a_{n-2} \log 2} \quad\left(2^{n-1} \leqslant x \leqslant 2^{n}\right)
$$

for $n \in \mathbf{N}$. We see immediately that the function $\lambda$ is positive, continuous, increasing, and unbounded on $(1, \infty)$. Further $2^{n-1} \leqslant x \leqslant 2^{n}$ implies that

$$
\frac{1}{a_{n-2}} \leqslant \lambda(x) \leqslant \frac{1}{a_{n-1}} \leqslant \chi\left(2^{n-1}\right) \leqslant \chi(x) .
$$

Since this leads to the inequality

$$
\int_{2^{n-1}}^{2^{n}} \frac{d x}{x \lambda(x)} \leqslant a_{n-2} \int_{2^{n-1}}^{2^{n}} \frac{d x}{x}=a_{n-2} \log 2
$$

(4.2) follows.

Finally we need to show that if

$$
E(x)=\frac{\lambda(x) \log 2}{(\log x)^{2}} \quad(x \geqslant 1)
$$

then the function $E$ is decreasing. This is easily verified by noting that

$$
\begin{aligned}
E^{\prime}(x) & =\frac{\left(a_{n-1}-a_{n-2}\right) \log x-2\left(n a_{n-1}-(n-1) a_{n-2}\right) \log 2}{a_{n-1} a_{n-2} x(\log x)^{3}} \\
& \leqslant \frac{(\log 2)\left((n-1) a_{n-2}-(n+1) a_{n-1}\right)}{a_{n-1} a_{n-2} x(\log x)^{3}} \leqslant 0
\end{aligned}
$$

when $2^{n-1}<x<2^{n}$. This completes the proof of Lemma 1 and, therefore, the proof of Theorem 2. Corollary 1 is readily deduced from Theorem 2.

\section{REFERENCES}

1. W. K. Hayman and B. Korenblum, A critical growth rate for functions regular in a disk, Michigan Math. J. 27 (1980), 21-30.

2. C. N. Linden, Functions regular in the unit circle, Proc. Cambridge Philos. Soc. 52 (1956), 49-60.

3. E. C. Titchmarsh, The theory of functions, Oxford Univ. Press, London, 1939.

Department of Pure Mathematics, University College of Swansea, University of Wales, SWANSEA, WALES SA28PP 